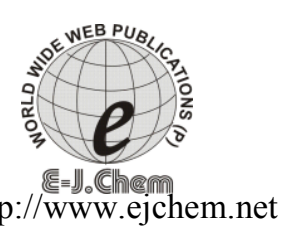

ISSN: 0973-4945; CODEN ECJHAO

E-Journal of Chemistry

2012, 9(4), 1897-1905

\title{
A Facile Synthesis and Studies of Some New Chalcones and Their Derivatives Based on Heterocyclic Ring
}

\author{
A. SOLANKEE*, K. PATEL, AND R. PATEL \\ Department of Chemistry, B.K.M. Science College, Valsad-396001, India. \\ dranjani_solankee@yahoo.com
}

Received 01 November 2011; Accepted 07 January 2012

\begin{abstract}
Chalcones (6a-f) have been prepared by the condensation of ketone (5) and different aromatic and heterocyclic aldehydes. These chalcones (6a-f) on treatment with guanidine hydrochloride and phenyl hydrazine hydrochloride in presence of alkali give aminopyrimidines (7a-f) and phenylpyrazolines (8a-f) respectively. All the newly synthesized compounds have been characterized on the basis of IR, ${ }^{1} \mathrm{H}$ NMR spectral data as well as physical data. Antibacterial activity (minimum inhibitory concentration MIC) against Gram-positive $S$. aureus MTCC 96 and S. pyogeneus MTCC 442 and Gram-negative $P$. aeruginosa MTCC 1688 and E. coli MTCC 443 bacteria, as well as antifungal acivity (MIC) against $C$. albicans MTCC 227, A. niger MTCC 282 and A. clavatus MTCC 1323 were determined by broth dilution method.
\end{abstract}

Keywords: Chalcones, aminopyrimidines, phenyl pyrazolines, spectral data, antimicrobial activity.

\section{Introduction}

Commonly $\alpha, \beta$ - unsaturated ketone is known as chalcone. Chalcones ${ }^{1,2}$ are very reactive compounds and increase their reactivity due to keto-ethylinic type of conjugated double bond system present in the molecule. Chalcones have occupied unique place in medicinal and biological chemistry due to their diverse pharmacological properties such as anticancer ${ }^{3}$, anti-inflammatory $^{4}$, antiviral ${ }^{5}$ and antibacterial ${ }^{6}$ etc.... Five membered heterocyclic like pyrazolines have found wide application as pharmaceutical and agrochemical agents. Pyrazoline derivatives have been found to possess wide range of therapeutic activities such as diuretic ${ }^{7}$, antifungal ${ }^{8}$, analgesic ${ }^{9}$, and mollucicidal ${ }^{10}$ etc.... Pyrimidine derivatives play a vital role in many biological processes and in synthesis of many drugs. Many pyrimidine derivatives have displayed diverse biological activities such as antitumor ${ }^{11}$, hypnotensive ${ }^{12}$, antiulcer $^{13}$, and anticonvulsant ${ }^{14}$ etc... 
In view of the above and in a continuation of our work ${ }^{15-17}$ on chalcones and its derivatives, we herein report a new series of chalcones (6a-f), aminopyrimidines (7a-f), and phenylpyrazolines (8a-f).

\section{Experimental}

All melting points were determined in open capillary and are uncorrected. The IR spectra were recorded on a FTIR - 8400 spectrometer. ${ }^{1} \mathrm{H}$ NMR spectra on a Bruker Avance DPX $400 \mathrm{MHz}$ spectrometer with $\mathrm{CDCl}_{3}$ as a solvent and tetramethylsilane (TMS) as internal standard. The chemical shifts are expressed in parts per million ( $\mathrm{ppm}$ ) downfield from the internal standard and signals are quoted as $s$ (singlet) and $m$ (multiplate). Analytical separation was conducted with silica Gel $60 \mathrm{~F}-254$ (Merck) plates of $0.25 \mathrm{~mm}$ thickness eluted with toluene : acetone $(10: 2 \mathrm{v} / \mathrm{v})$ and were visualized with UV $(254 \mathrm{~nm})$ or iodine to check the purity of the synthesized compounds.

\section{Preparation of 2-(2', 4'-difluorophenylamino)-4, 6-dichloro-s-triazine (3)}

2, 4-Difluoroaniline $(0.01 \mathrm{~mol}, 1.29 \mathrm{~g}$ in $10 \mathrm{~mL}$ acetone) was added slowly to cyanuric chloride $(0.01 \mathrm{~mol}, 1.845 \mathrm{~g}$ in $30 \mathrm{~mL}$ acetone $)$ with constant stirring for 4 hours at 0 to $5^{0} \mathrm{C}$. Periodically, sodium carbonate solution $(0.005 \mathrm{~mol}, 0.53 \mathrm{~g}$ in $10 \mathrm{~mL}$ water $)$ was added drop wise to neutralized $\mathrm{HCl}$ evolved during the reaction. The progress of the reaction was monitored on TLC plate. Finally, the content was poured into crushed ice. The solid seprated out was filtered, washed with water, dried and recrystallised from alcohol to give (3).

\section{Preparation of 2, 4-bis-(2', 4'-difluorophenylamino)-6-chloro-s-triazine (4)}

2-4-Difluoroaniline $(0.01 \mathrm{~mol}, 1.29 \mathrm{~g}$ in $10 \mathrm{~mL}$ acetone) was added slowly to compound (3) ( $0.01 \mathrm{~mol}, 2.77 \mathrm{~g}$ in $35 \mathrm{~mL}$ acetone) with constant stirring for 6 hours at room temperature. Periodically, sodium carbonate solution $(0.005 \mathrm{~mol}, 0.53 \mathrm{~g}$ in $10 \mathrm{~mL}$ water $)$ was added drop wise to neutralized $\mathrm{HCl}$ evolved during the reaction. The progress of the reaction was monitored on TLC plate. Finally, the content was poured into crushed ice. The solid separated out was filtered, washed with water, dried and recrystallized from alcohol to give (4).

Preparation of 2,4-bis-(2',4'-difluorophenylamino)-6-(4'-acetylphenylamino)-s-triazine

4-Aminoacetophenone ( $0.01 \mathrm{~mol})$ and compound (4) $(0.01 \mathrm{~mol})$ were dissolved in acetone $(40 \mathrm{~mL})$. The reaction mixture was refluxed for 8 hours. Periodically, sodium carbonate solution $(0.005 \mathrm{~mol}, 0.53 \mathrm{~g}$ in $10 \mathrm{~mL}$ water $)$ was added to neutralized $\mathrm{HCl}$ evolved during the reaction. The progress of the reaction was monitored on TLC plate. After completion the reaction the content was poured into crushed ice. The solid separated out was filtered, washed with water, dried and recrystallised from alcohol to give (5).

Preparation of 2,4-bis-(2',4'-difluorophenylamino)-6-[4'-\{5"-(3"'-methoxyphenyl)-2"propenon-1"-yl? phenyl amino]-s-triazine (6a)

Compound (5) $(0.01 \mathrm{~mol})$ was dissolved in DMF $(30 \mathrm{~mL})$ and 3-methoxybenzaldehyde $(0.01 \mathrm{~mol})$ was added to it. Then solution of $\mathrm{KOH}(5 \mathrm{~mL}$ of $40 \%)$ was added to the reaction mixture with constant stirring at room temperature. The progress of the reaction was monitored on TLC plate. After completion the reaction mixture was poured into crushed ice 
and neutralised with $\mathrm{HCl}$. The product separated out was filtered, washed with water, dried and recrystallised from alcohol.

Similarly the remaining compounds (6b-f) were prepared by this method.

Compound (6a)

IR $\left(\mathrm{KBr}, \mathrm{cm}^{-1}\right)$ : 3303 (N-H str.), 3072 (=CH str.), 1650 (C=O str.), 1508 (C=C str.), 1023 (C-O-C str.), 808 (C-N str., $s$-triazine moiety), $1085 \mathrm{~cm}^{-1}$ (C-F str.) ; ${ }^{1} \mathrm{H}$ NMR $\left(\mathrm{CDCl}_{3}, \delta\right.$, ppm) : $3.80\left(3 \mathrm{H}, s, \mathrm{~m}-\mathrm{OCH}_{3}\right), 6.41(1 \mathrm{H}, \mathrm{d},-\mathrm{CO}-\mathrm{CH}=), 8.03(1 \mathrm{H}, \mathrm{d}, \mathrm{Ar}-\mathrm{CH}=), 6.98-7.90$ $\left(17 \mathrm{H}, m\right.$, Ar-H and -NH). Anal. Calcd. For $\mathrm{C}_{31} \mathrm{H}_{22} \mathrm{~F}_{4} \mathrm{~N}_{6} \mathrm{O}_{2}$ : C, 63.48; H, 3.78; N, 14.33 . Found: C: $63.50 ; \mathrm{H}: 3.80 ; \mathrm{N}: 14.35 \%$.

\section{Compound (6b)}

IR $\left(\mathrm{KBr}, \mathrm{cm}^{-1}\right)$ : 3305 (N-H str.), 3075 (=CH str.), 1650 (C=O str.), 1517 (C=C str.), 1020 (C-O-C str.), 812 (C-N str., $s$-triazine moiety), $1082 \mathrm{~cm}^{-1}$ (C-F str.); ${ }^{1} \mathrm{H}$ NMR $\left(\mathrm{CDCl}_{3}, \delta\right.$, ppm) : $3.80\left(6 \mathrm{H}, s, \mathrm{~m}-\mathrm{OCH}_{3}\right), 3.83\left(3 \mathrm{H}, s, \mathrm{p}-\mathrm{OCH}_{3}\right), 6.41(1 \mathrm{H}, \mathrm{d},-\mathrm{CO}-\mathrm{CH}=), 8.05(1 \mathrm{H}, \mathrm{d}$, $\mathrm{Ar}-\mathrm{CH}=), 6.98-7.92(15 \mathrm{H}, m, \mathrm{Ar}-\mathrm{H}$ and $-\mathrm{NH})$. Anal. Calcd. For $\mathrm{C}_{33} \mathrm{H}_{26} \mathrm{~F}_{4} \mathrm{~N}_{6} \mathrm{O}_{4}: \mathrm{C}, 61.30$; H, 4.05; N, 13.00. Found: C: $61.28 ; \mathrm{H}: 4.01 ; \mathrm{N}: 12.98 \%$.

\section{Compound (6c)}

IR $\left(\mathrm{KBr}, \mathrm{cm}^{-1}\right)$ : 3303 (N-H str.), 3077 (=CH str.), 1652 (C=O str.), 1510 (C=C str.), 1030 (C-O-C str.), 814 (C-N str., $s$-triazine moiety), $1083 \mathrm{~cm}^{-1}$ (C-F str.) ; ${ }^{1} \mathrm{H}$ NMR $\left(\mathrm{CDCl}_{3}, \delta\right.$, ppm) : $6.39(1 \mathrm{H}, \mathrm{d},-\mathrm{CO}-\mathrm{CH}=), 8.03(1 \mathrm{H}, \mathrm{d}, \mathrm{Ar}-\mathrm{CH}=), 6.98-7.91(22 \mathrm{H}, m, \mathrm{Ar}-\mathrm{H}$ and $\mathrm{NH})$. Anal. Calcd. For $\mathrm{C}_{36} \mathrm{H}_{24} \mathrm{~F}_{4} \mathrm{~N}_{6} \mathrm{O}_{2}$ : C, 66.66; H, 3.73; N, 12.96. Found: C: 66.65; H: 3.70 ; N: $12.94 \%$.

\section{Compound (6d)}

IR (KBr,cm $\left.{ }^{-1}\right)$ : 3304 (N-H str.), 3070 (=CH str.), 1651 ( $\mathrm{C}=\mathrm{O}$ str. $), 1515$ (C=C str.), 1545 $\left(\mathrm{C}-\mathrm{NO}_{2}\right), 818$ (C-N str., $s$-triazine moiety), $1084 \mathrm{~cm}^{-1}$ (C-F str.) $;{ }^{1} \mathrm{H} \mathrm{NMR}\left(\mathrm{CDCl}_{3}, \delta, \mathrm{ppm}\right)$ : $6.41(1 \mathrm{H}, \mathrm{d},-\mathrm{CO}-\mathrm{CH}=), 8.03(1 \mathrm{H}, \mathrm{d}, \mathrm{Ar}-\mathrm{CH}=), 6.95-7.91(17 \mathrm{H}, m, \mathrm{Ar}-\mathrm{H}$ and $-\mathrm{NH})$. Anal. Calcd. For $\mathrm{C}_{30} \mathrm{H}_{19} \mathrm{~F}_{4} \mathrm{~N}_{7} \mathrm{O}_{3}$ : C, 59.90; H, 3.18; N, 16.30. Found : C: 59.87; H: 3.17; N: $16.28 \%$.

\section{Compound (6e)}

IR $\left(\mathrm{KBr}, \mathrm{cm}^{-1}\right)$ : 3310 (N-H str.), 3060 (=CH str.), 1653 (C=O str.), 1505 (C=C str.), 820 (C-N str., $s$-triazine moiety), 595 (C-Br), $1087 \mathrm{~cm}^{-1}$ (C-F str.) ; ${ }^{1} \mathrm{H}$ NMR $\left(\mathrm{CDCl}_{3}, \delta\right.$, ppm): $6.44(1 \mathrm{H}, \mathrm{d},-\mathrm{CO}-\mathrm{CH}=), 8.02(1 \mathrm{H}, \mathrm{d}, \mathrm{Ar}-\mathrm{CH}=), 6.95-7.91(17 \mathrm{H}, m, \mathrm{Ar}-\mathrm{H}$ and $-\mathrm{NH})$. Anal. Calcd. For $\mathrm{C}_{30} \mathrm{H}_{19} \mathrm{~F}_{4} \mathrm{~N}_{6} \mathrm{OBr}$ : C, 56.71; H, 3.01; N, 13.23. Found: $\mathrm{C}: 56.70 ; \mathrm{H}: 3.00 ; \mathrm{N}$ : $13.22 \%$.

\section{Compound (6f)}

IR $\left(\mathrm{KBr}, \mathrm{cm}^{-1}\right): 3308$ (N-H str.), 3070 (=CH str.), 1655 (C=O str.), 1517 (C=C str.), 1035 (C-O-C str.), 810 (C-N str., $s$-triazine moiety), $801 \mathrm{~cm}^{-1}$ (C-Cl str.) ; ${ }^{1} \mathrm{H} \mathrm{NMR}\left(\mathrm{CDCl}_{3}, \delta\right.$, ppm): $6.43(1 \mathrm{H}, \mathrm{d},-\mathrm{CO}-\mathrm{CH}=), 8.03(1 \mathrm{H}, \mathrm{d}, \mathrm{Ar}-\mathrm{CH}=), 6.95-7.91(16 \mathrm{H}, m, \mathrm{Ar}-\mathrm{H}$ and $\mathrm{NH}$ ). Anal. Calcd. For $\mathrm{C}_{28} \mathrm{H}_{18} \mathrm{~F}_{4} \mathrm{~N}_{6} \mathrm{O}_{2}$ : C, 61.54; H, 3.32; N, 15.38. Found: C: 61.50; H: 3.32 ; N: $15.35 \%$.

Preparation of 2,4-bis-(2',4'-difluorophenylamino)-6-[4'-\{2"-amino-6"-(3"'methoxyphenyl)- pyrimidin -4 "-ylsphenylamino]-s-triazine (7a) 
Compound 6a $(0.01 \mathrm{~mol})$ was dissolved in ethyl alcohol $(25 \mathrm{~mL})$ and guanidine hydrochloride $(0.01 \mathrm{~mol})$ was added to it. Then solution of $\mathrm{KOH}(5 \mathrm{~mL}$ of $40 \%)$ was added to the reaction mixture and refluxed for 8 hours. The progress of the reaction was monitored on TLC plate. After completion the reaction mixture was cooled and poured into crushed ice and neutralised with dilute $\mathrm{HCl}$. The product separated out was filtered, washed with water, dried and recrystallised from alcohol to give $7 \mathrm{a}$.

Similarly the remaining compounds (7b-f) were prepared by this method.

\section{Compound (7a)}

IR (KBr,cm $\left.{ }^{-1}\right)$ : 3405 (N-H str.), 3065 (=CH str.), 809 (C-N str., $s$-triazine moiety), 825 ( C$\mathrm{H}$ bending), 1645 ( $\mathrm{C}=\mathrm{N}$ str, pyrimidine moiety), 1266 (C-O-C str.), 1095 (C-F str.) ; ${ }^{1} \mathrm{H}$ NMR $\left(\mathrm{CDCl}_{3}, \delta, \mathrm{ppm}\right): 3.81\left(3 \mathrm{H}, s, \mathrm{~m}-\mathrm{OCH}_{3}\right), 5.1\left(2 \mathrm{H}, s,-\mathrm{NH}_{2}\right), 6.85(1 \mathrm{H}, s,-\mathrm{CH}=), 7.0-$ $8.0(17 \mathrm{H}, m, \mathrm{Ar}-\mathrm{H}$ and $-\mathrm{NH})$. Anal. Calcd. For $\mathrm{C}_{32} \mathrm{H}_{23} \mathrm{~F}_{4} \mathrm{~N}_{9} \mathrm{O}: \mathrm{C}, 61.44 ; \mathrm{H}, 3.71 ; \mathrm{N}, 20.15$. Found : C: $61.42 ; \mathrm{H}: 3.70 ; \mathrm{N}: 20.10 \%$.

\section{Compound (7b)}

IR $\left(\mathrm{KBr}, \mathrm{cm}^{-1}\right)$ : 3410 (N-H str.), 3070 (=CH str.), 810 (C-N str, s-triazine moiety), 830 (C-H bending), 1642 ( $\mathrm{C}=\mathrm{N}$ str, pyrimidine moiety), 1267 (C-O-C str), 1098 (C-F str) ; ${ }^{1} \mathrm{H}$ NMR $\left(\mathrm{CDCl}_{3}, \delta, \mathrm{ppm}\right): 3.81\left(6 \mathrm{H}, s, \mathrm{~m}-\mathrm{OCH}_{3}\right), 3.85\left(3 \mathrm{H}, s, \mathrm{p}-\mathrm{OCH}_{3}\right), 5.2\left(2 \mathrm{H}, s,-\mathrm{NH}_{2}\right), 6.87(1 \mathrm{H}$, $s,-\mathrm{CH}=), 7.0-8.0(15 \mathrm{H}, m, \mathrm{Ar}-\mathrm{H}$ and $-\mathrm{NH})$. Anal. Calcd. For $\mathrm{C}_{34} \mathrm{H}_{27} \mathrm{~F}_{4} \mathrm{~N}_{9} \mathrm{O}_{3}: \mathrm{C}, 59.56 ; \mathrm{H}$, 3.97 ; N, 18.39. Found: C, 59.52; H, 3.95; N, 18.35\%.

\section{Compound (7c)}

IR $\left(\mathrm{KBr}, \mathrm{cm}^{-1}\right): 3412$ (N-H str.), 3072 (=CH str.), 812 (C-N str., s-triazine moiety), 832 (C-H bending), 1641 ( $\mathrm{C}=\mathrm{N}$ str, pyrimidine moiety), 1270 (C-O-C str.), 1098 (C-F str.); ${ }^{1} \mathrm{H}$ NMR $\left(\mathrm{CDCl}_{3}, \delta, \mathrm{ppm}\right): 5.3\left(2 \mathrm{H}, s,-\mathrm{NH}_{2}\right), 6.83(1 \mathrm{H}, s,-\mathrm{CH}=), 7.0-8.0(22 \mathrm{H}, m, \mathrm{Ar}-\mathrm{H}$ and $-\mathrm{NH})$. Anal. Calcd. For $\mathrm{C}_{37} \mathrm{H}_{25} \mathrm{~F}_{4} \mathrm{~N}_{9} \mathrm{O}: \mathrm{C}, 64.63 ; \mathrm{H}, 3.66 ; \mathrm{N}, 18.33$. Found: C: 64.60; H, 3.64; N, $18.30 \%$.

\section{Compound (7d)}

IR $\left(\mathrm{KBr}, \mathrm{cm}^{-1}\right)$ : 3410 (N-H str.), 3071 (=CH str.), 815 (C-N str, s-triazine moiety), 830 (C-H bending), $1550\left(\mathrm{C}-\mathrm{NO}_{2}\right), 1644\left(\mathrm{C}=\mathrm{N}\right.$ str, pyrimidine- moiety), 1087 (C-F str.) ; ${ }^{1} \mathrm{H}$ NMR $\left(\mathrm{CDCl}_{3}, \delta, \mathrm{ppm}\right): 5.1\left(2 \mathrm{H}, s,-\mathrm{NH}_{2}\right), 6.81(1 \mathrm{H}, s,-\mathrm{CH}=), 7.0-8.0(17 \mathrm{H}, m, \mathrm{Ar}-\mathrm{H}$ and $-\mathrm{NH})$. Anal. Calcd. For $\mathrm{C}_{31} \mathrm{H}_{20} \mathrm{~F}_{4} \mathrm{~N}_{10} \mathrm{O}_{2}$ : C, 58.13; H, 3.15; N, 21.87. Found: C: 58.11; H: 3.12; N: $21.85 \%$.

\section{Compound (7e)}

IR $\left(\mathrm{KBr}, \mathrm{cm}^{-1}\right)$ : 3410 (N-H str.), 3072 (=CH str.), 816 (C-N str, s-triazine moiety), 832 ( C-H bending), $1646\left(\mathrm{C}=\mathrm{N}\right.$ str, pyrimidinemoiety), 605 (C-Br), 1087 (C-F str.) ; ${ }^{1} \mathrm{H}$ NMR $\left(\mathrm{CDCl}_{3}, \delta, \mathrm{ppm}\right): 5.2\left(2 \mathrm{H}, s,-\mathrm{NH}_{2}\right), 6.82(1 \mathrm{H}, s,-\mathrm{CH}=), 7.1-8.1(17 \mathrm{H}, m, \mathrm{Ar}-\mathrm{H}$ and $-\mathrm{NH})$. Anal. Calcd. For $\mathrm{C}_{31} \mathrm{H}_{20} \mathrm{~F}_{4} \mathrm{~N}_{9} \mathrm{Br}: \mathrm{C}, 55.21 ; \mathrm{H}, 2.99 ; \mathrm{N}, 18.69$. Found : C: 55.19; H, 2.95; N, $18.65 \%$.

\section{Compound (7f)}

IR $\left(\mathrm{KBr}, \mathrm{cm}^{-1}\right)$ : 3413 (N-H str.), 3074 (=CH str.), 818 (C-N str, $s$-triazine moiety), 835 ( C-H bending), 1643 ( $\mathrm{C}=\mathrm{N}$ str, pyrimidine moiety), 1271 (C-O-C str.), 1087 (C-F str.); ${ }^{1} \mathrm{H}$ NMR $\left(\mathrm{CDCl}_{3}, \delta, \mathrm{ppm}\right): 5.3\left(2 \mathrm{H}, s,-\mathrm{NH}_{2}\right), 6.85(1 \mathrm{H}, s,-\mathrm{CH}=), 7.1-8.1(16 \mathrm{H}, m, \mathrm{Ar}-\mathrm{H}$ and $-\mathrm{NH})$. Anal. Calcd. For $\mathrm{C}_{29} \mathrm{H}_{19} \mathrm{~F}_{4} \mathrm{~N}_{9} \mathrm{O}: \mathrm{C}, 59.49 ; \mathrm{H}, 3.27 ; \mathrm{N}, 21.53$. Found: C: 59.45; H, 3.26; N, $21.50 \%$.

Preparation of 2,4-bis-(2',4'-difluorophenylamino)-6--[4'-\{1"-phenyl-5"-(3'"-methoxyphenyl)-2"-pyrazolin-3"-yl? phenylamino]-s-triazine (8a) 
Compound 6a $(0.01 \mathrm{~mol})$ was dissolved in alcohol $(25 \mathrm{~mL})$ and phenyl hydrazine hydrochloride $(0.01 \mathrm{~mol}, 1.44 \mathrm{~g})$ in $40 \mathrm{~mL}$ alcohol was refluxed for 10 hours in the presence of $40 \% \mathrm{KOH}(3 \mathrm{~mL})$. The progress of the reaction was monitored on TLC plate. After completion the reaction mixture was cooled and poured into crushed ice and neutralized with dilute $\mathrm{HCl}$. The product separated out was filtered, washed with water, dried and recrystallised from alcohol to give $8 \mathrm{a}$.

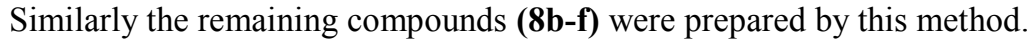

\section{Compound (8a)}

IR (KBr,cm $\left.{ }^{-1}\right)$ : 3402 (N-H str.), 3060 (=CH str.), 809 (C-N str, s-triazine moiety), 1574 (C=N str, pyrazoline moiety), 2985 (C-H str, pyrazoline moiety ), 1095 (C-F str.) ; ${ }^{\mathrm{H}} \mathrm{NMR}$ $\left(\mathrm{CDCl}_{3}, \delta, \mathrm{ppm}\right): 3.0(1 \mathrm{H}, d d,-\mathrm{CHa}-\mathrm{CH}), 3.2(1 \mathrm{H}, d d,-\mathrm{CHb}-\mathrm{CH}), 3.8\left(3 \mathrm{H}, s, \mathrm{~m}-\mathrm{OCH}_{3}\right)$, $7.0-8.0(22 \mathrm{H}, m$, Ar-H and $-\mathrm{NH}), 5.3\left(1 \mathrm{H}, t,-\mathrm{CH}-\mathrm{CH}_{2}-\mathrm{Ar}-\right)$. Anal. Calcd. For $\mathrm{C}_{37} \mathrm{H}_{28} \mathrm{~F}_{4} \mathrm{~N}_{8} \mathrm{O}: \mathrm{C}, 65.68 ; \mathrm{H}, 4.17$; N, 16.56. Found: C: $65.65 ; \mathrm{H}: 4.15 ; \mathrm{N}: 16.50 \%$.

\section{Compound (8b)}

IR $\left(\mathrm{KBr}, \mathrm{cm}^{-1}\right)$ : 3404 (N-H str.), 3065 (=CH str.), 811 (C-N str, s-triazine moiety), 1575 (C=N str., pyrazoline moiety), 2987 (C-H str., pyrazoline moiety ), 1270 (C-O-C str.), 1090 (C-F str.) ; ${ }^{1} \mathrm{H}$ NMR $\left(\mathrm{CDCl}_{3}, \delta, \mathrm{ppm}\right): 3.1(1 \mathrm{H}, d d,-\mathrm{CHa}-\mathrm{CH}), 3.3(1 \mathrm{H}, d d,-\mathrm{CHb}-\mathrm{CH}), 3.4$ $\left(6 \mathrm{H}, s, \mathrm{~m}-\mathrm{OCH}_{3}\right), 3.6\left(3 \mathrm{H}, s, \mathrm{p}-\mathrm{OCH}_{3}\right), 7.0-8.0(21 \mathrm{H}, m, \mathrm{Ar}-\mathrm{H}$ and $-\mathrm{NH}), 5.2(1 \mathrm{H}, t,-\mathrm{CH}-$ $\mathrm{CH}_{2}$-Ar-). Anal. Calcd. For $\mathrm{C}_{39} \mathrm{H}_{32} \mathrm{~F}_{4} \mathrm{~N}_{8} \mathrm{O}_{3}: \mathrm{C}, 63.58 ; \mathrm{H}, 4.38 ; \mathrm{N}, 15.21$. Found: C: 63.56; $\mathrm{H}, 4.35 ; \mathrm{N}, 15.18 \%$.

\section{Compound (8c)}

IR (KBr,cm ${ }^{-1}$ ): 3405 (N-H str.), 3065 (=CH str.), 810 (C-N str, $s$-triazine moiety), 1575 (C=N str, pyrazoline moiety), 2983 (C-H str, pyrazoline moiety ), 1097 (C-F str.) ; ${ }^{1} \mathrm{H}$ NMR $\left(\mathrm{CDCl}_{3}, \delta, \mathrm{ppm}\right): 3.2(1 \mathrm{H}, d d,-\mathrm{CHa}-\mathrm{CH}), 3.3(1 \mathrm{H}, d d,-\mathrm{CHb}-\mathrm{CH}), 7.0-8.0(27 \mathrm{H}, m, \mathrm{Ar}-$ $\mathrm{H}$ and $-\mathrm{NH}), 5.4\left(1 \mathrm{H}, t,-\mathrm{CH}-\mathrm{CH}_{2}-\mathrm{Ar}-\right)$. Anal. Calcd. For $\mathrm{C}_{42} \mathrm{H}_{30} \mathrm{~F}_{4} \mathrm{~N}_{8} \mathrm{O}: \mathrm{C}, 68.29 ; \mathrm{H}, 4.09$; N, 15.17. Found: C: $68.26 ; \mathrm{H}, 4.05 ; \mathrm{N}, 15.15 \%$.

\section{Compound (8d)}

IR $\left(\mathrm{KBr}, \mathrm{cm}^{-1}\right)$ : 3406 (N-H str.), 3066 (=CH str.), 809 (C-N str, s-triazine moiety), 1540 (C$\mathrm{NO}_{2}$ ), $1578(\mathrm{C}=\mathrm{N}$ str, pyrazoline moiety), 2987 (C-H str, pyrazoline moiety ), 1096 (C-F str.) ; ${ }^{1} \mathrm{H}$ NMR $\left(\mathrm{CDCl}_{3}, \delta, \mathrm{ppm}\right): 3.0(1 \mathrm{H}, d d,-\mathrm{CHa}-\mathrm{CH}), 3.3(1 \mathrm{H}, d d,-\mathrm{CHb}-\mathrm{CH}), 7.1-$ $8.1(22 \mathrm{H}, m, \mathrm{Ar}-\mathrm{H}$ and $-\mathrm{NH}), 5.5\left(1 \mathrm{H}, t,-\mathrm{CH}-\mathrm{CH}_{2}-\mathrm{Ar}-\right)$. Anal. Calcd. For $\mathrm{C}_{36} \mathrm{H}_{25} \mathrm{~F}_{4} \mathrm{~N}_{9} \mathrm{O}_{2}: \mathrm{C}$, 62.52; H, 3.64; N, 18.23. Found: C: 62.50; H: 3.62; N: $18.20 \%$.

\section{Compound (8e)}

IR $\left(\mathrm{KBr}, \mathrm{cm}^{-1}\right)$ : 3407 (N-H str.), 3070 (=CH str.), 813 (C-N str, $s$-triazine moiety), 1580 $(\mathrm{C}=\mathrm{N}$ str, pyrazoline moiety), 2987 (C-H str, pyrazoline moiety ), 1098 (C-F str.), 595 (C$\mathrm{Br}) ;{ }^{1} \mathrm{H}$ NMR $\left(\mathrm{CDCl}_{3}, \delta, \mathrm{ppm}\right): 3.1(1 \mathrm{H}, d d,-\mathrm{CHa}-\mathrm{CH}), 3.3(1 \mathrm{H}, d d,-\mathrm{CHb}-\mathrm{CH}), 7.2-8.2$ $(22 \mathrm{H}, m, \mathrm{Ar}-\mathrm{H}$ and $-\mathrm{NH}), 5.5\left(1 \mathrm{H}, t,-\mathrm{CH}-\mathrm{CH}_{2}-\mathrm{Ar}-\right)$. Anal. Calcd. For $\mathrm{C}_{36} \mathrm{H}_{25} \mathrm{~F}_{4} \mathrm{~N}_{8} \mathrm{Br}: \mathrm{C}$, 59.60; H, 3.47; N, 15.44. Found: C: 59.58; H, 3.45; N, 15.42\%.

\section{Compound (8f)}

IR (KBr,cm $\left.{ }^{-1}\right)$ : 3408 (N-H str.), 3068 (=CH str.), 811 (C-N str, s-triazine moiety), $1578(\mathrm{C}=\mathrm{N}$ str, pyrazoline moiety), 2987 (C-H str, pyrazoline moiety ), 1265 (C-O-C str.), 1096 (C-F str.); ${ }^{1} \mathrm{H}$ NMR $\left(\mathrm{CDCl}_{3}, \delta, \mathrm{ppm}\right): 3.0(1 \mathrm{H}, d d,-\mathrm{CHa}-\mathrm{CH}), 3.1(1 \mathrm{H}, d d$, $\mathrm{CHb}-\mathrm{CH}), 7.3-8.3(21 \mathrm{H}, m, \mathrm{Ar}-\mathrm{H}$ and $-\mathrm{NH}), 5.5\left(1 \mathrm{H}, t,-\mathrm{CH}-\mathrm{CH}_{2}-\mathrm{Ar}-\right)$. Anal. Calcd. For $\mathrm{C}_{34} \mathrm{H}_{24} \mathrm{~F}_{4} \mathrm{~N}_{8} \mathrm{O}: \mathrm{C}, 64.15 ; \mathrm{H}, 3.80 ; \mathrm{N}, 17.60$. Found: C: $64.12 ; \mathrm{H}, 3.78 ; \mathrm{N}, 17.58 \%$. 


\section{Results and Discussion}

The in vitro antibacterial and antifungal activities of the compounds are shown in (Table 2). The MICs $(\mu \mathrm{g} / \mathrm{mL})$ were carried out by broth microdilution method as described by Rattan ${ }^{18}$. The newly synthesised compounds (6a-f), (7a-f) and (8a-f) were screened for their antibacterial activity against two Gram positive bacteria srains: $S$. aureus MTCC96 and $S$. pyogenes MTCC442 and two Gram negative bacteria E.coli MTCC443 and P. aeruginosa MTCC1688 and compared with standard drug: Ampicillin and Antifungal activity against C. albicans MTCC227, A. niger MTCC282 and A. clavatus MTCC1323 organisms was determined by same method and compared with standard drug: Griseofulvin.
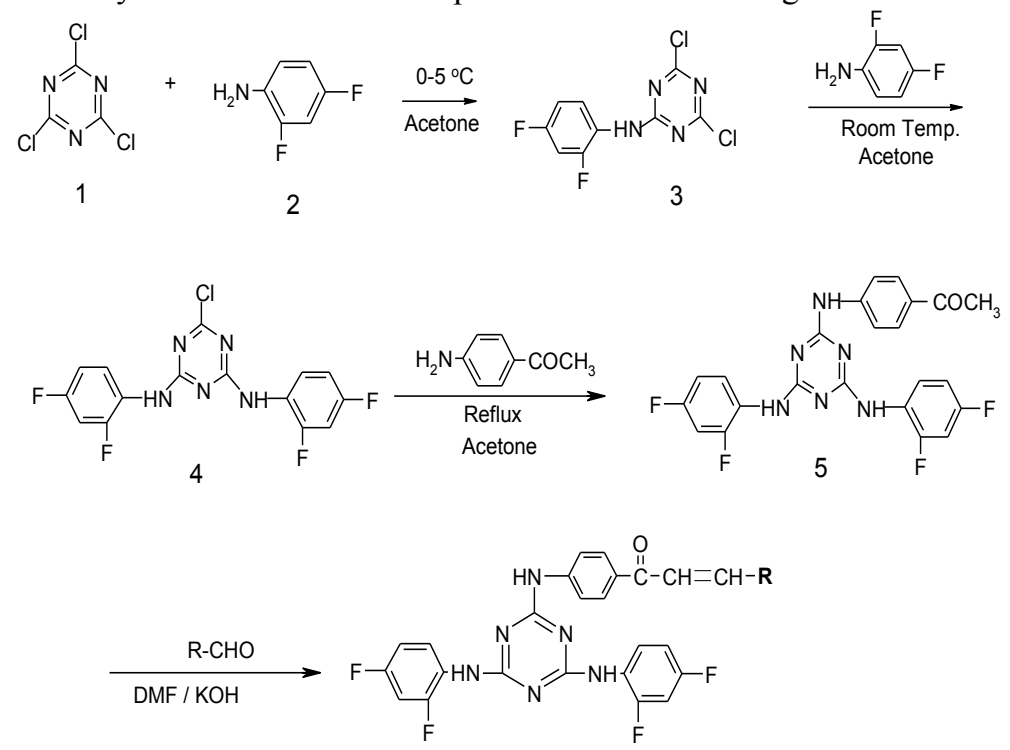

6a-f

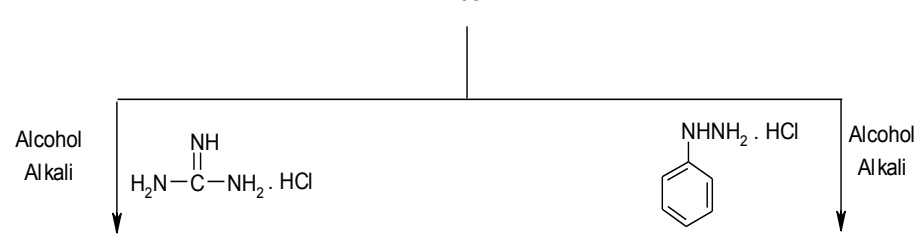<smiles>[R]c1cc(-c2ccc(Nc3nc(N)nc(Nc4cccc(F)c4)n3)cc2)nc(N)n1</smiles>

$7 a-f$<smiles>[R]C1CC(c2ccc(Nc3nc(Nc4cccc(F)c4)nc(Nc4ccc(F)cc4F)n3)cc2)=NN1c1ccccc1</smiles>

$8 a-f$ 
Table 1. Characterization data of compounds (6a-f), (7a-f) and (8a-f).

\begin{tabular}{|c|c|c|c|}
\hline Compds & $\mathrm{R}$ & M.P. ${ }^{\circ} \mathrm{C}$ & $\%$ Yield \\
\hline $6 a$ & 3-Methoxyphenyl & 99 & 72 \\
\hline $6 b$ & 3,4,5-Trimethoxyphenyl & 112 & 69 \\
\hline $6 c$ & 3-Phenoxyphenyl & 103 & 76 \\
\hline $6 \mathrm{~d}$ & 3-Nitrophenyl & 153 & 69 \\
\hline $6 e$ & 3-Bromophenyl & 102 & 56 \\
\hline $6 f$ & 2-Furanyl & 113 & 72 \\
\hline $7 a$ & 3-Methoxyphenyl & 129 & 63 \\
\hline $7 b$ & 3,4,5-Trimethoxyphenyl & 122 & 71 \\
\hline $7 \mathrm{c}$ & 3-Phenoxyphenyl & 158 & 60 \\
\hline $7 d$ & 3-Nitrophenyl & 193 & 69 \\
\hline $7 e$ & 3-Bromophenyl & 180 & 67 \\
\hline $7 f$ & 2-Furanyl & 131 & 51 \\
\hline $8 \mathrm{a}$ & 3-Methoxyphenyl & 125 & 69 \\
\hline $8 b$ & 3,4,5-Trimethoxyphenyl & 185 & 66 \\
\hline $8 \mathrm{c}$ & 3-Phenoxyphenyl & 131 & 64 \\
\hline $8 \mathrm{~d}$ & 3-Nitrophenyl & 245 & 73 \\
\hline $8 \mathrm{e}$ & 3-Bromophenyl & 117 & 59 \\
\hline $8 \mathrm{f}$ & 2-Furanyl & 135 & 61 \\
\hline
\end{tabular}

\section{Antibacterial Activity}

From the screening results (Table -2 ), The result shows that compounds $\mathbf{6 c}, \mathbf{6 d}, \mathbf{6 e}, \mathbf{7 c}$, $\mathbf{8 a}, \mathbf{8 c}$ and $\mathbf{8 f}$ exhibited good activity $(25-125 \mu \mathrm{g} / \mathrm{mL})$ against $E$. coli ; compound 8e exhibited good activity $(50-100 \mu \mathrm{g} / \mathrm{mL})$ against $P$. aeruginosa . In Gram positive bacterial strains compounds $6 \mathbf{b}, \mathbf{6 f}, 7 \mathbf{a}, 8 \mathbf{b}, \mathbf{8 c}$ and $8 d$ showed good to very good activity $(25-150 \mu \mathrm{g} / \mathrm{mL})$ against $S$. aureus; whereas compound 7a showed good activity $(62.5-$ $100 \mu \mathrm{g} / \mathrm{mL}$ ) against $S$. pyogenes compared with Ampicillin. All others compounds show moderately active or less active against all bacterial strains.

\section{Antifungal Activity}

From the screening results (Table - 2), Compounds $6 \mathbf{a}, \mathbf{6 b}, \mathbf{7 b}, \mathbf{7 c}, \mathbf{7 e}, \mathbf{8 a}$ and $8 \mathbf{b}$ showed good activity against $C$. albicans compared with Griseofulvin, while compounds $\mathbf{6 f}$ and $\mathbf{8 c}$ showed very good activity against $C$. albicans.

\section{Conclusion}

Compounds having methoxy and phenoxy groups have exhibited more antimicrobial activity. These results suggest that the chalcone derivatives have excellent scope for further development as commercial antimicrobial agents. Further experiments were needed to elucidate their mechanism of action. 
Table 2. Antibacterial and antifungal activity data of compounds $6(a-f), 7(a-f)$ and $8(a-f)$.

\begin{tabular}{|c|c|c|c|c|c|c|c|}
\hline \multirow{3}{*}{ Comps } & \multicolumn{4}{|c|}{$\begin{array}{c}\text { Minimal bactericidal concentration } \\
\qquad \mu \mathrm{g} / \mathrm{mL}\end{array}$} & \multirow{2}{*}{\multicolumn{3}{|c|}{$\begin{array}{l}\text { Minimal fungicidal } \\
\text { concentration } \mu \mathrm{g} / \mathrm{mL}\end{array}$}} \\
\hline & \multicolumn{2}{|c|}{ Gram negative } & \multicolumn{2}{|c|}{ Gram positive } & & & \\
\hline & $\begin{array}{c}\text { E. coli } \\
\text { MTCC- } \\
443\end{array}$ & $\begin{array}{c}\text { P. } \\
\text { aerug } \\
\text { MTCC- } \\
1688\end{array}$ & $\begin{array}{c}\text { S. } \\
\text { aureus } \\
\text { MTCC- } \\
96\end{array}$ & $\begin{array}{c}\text { S. } \\
\text { pyogenus } \\
\text { MTCC- } \\
442\end{array}$ & $\begin{array}{c}\text { C. } \\
\text { albicans } \\
\text { MTCC- } \\
227\end{array}$ & $\begin{array}{c}\text { A. niger } \\
\text { MTCC- } \\
282\end{array}$ & $\begin{array}{c}\text { A. } \\
\text { clavatus } \\
\text { MTCC- } \\
1323\end{array}$ \\
\hline $6 a$ & 500 & 200 & 200 & 250 & 500 & 500 & 500 \\
\hline $6 \mathrm{~b}$ & 200 & 200 & 125 & 125 & 500 & $>1000$ & $>1000$ \\
\hline $6 c$ & 125 & 200 & 500 & 250 & 1000 & 250 & 500 \\
\hline $6 \mathrm{~d}$ & 125 & 250 & 250 & 250 & 1000 & 1000 & 1000 \\
\hline $6 e$ & 100 & 125 & 500 & 500 & 1000 & 500 & 1000 \\
\hline $6 \mathrm{f}$ & 500 & 250 & 100 & 125 & 200 & 1000 & 1000 \\
\hline $7^{\mathrm{a}}$ & 250 & 200 & 125 & 100 & 1000 & 500 & 500 \\
\hline $7 b$ & 200 & 125 & 250 & 250 & 500 & $>1000$ & $>1000$ \\
\hline $7 \mathrm{c}$ & 125 & 200 & 250 & 200 & 500 & $>1000$ & $>1000$ \\
\hline $7 \mathrm{~d}$ & 200 & 250 & 200 & 250 & 1000 & 500 & 500 \\
\hline $7 \mathrm{e}$ & 250 & 250 & 200 & 250 & 500 & 1000 & 1000 \\
\hline $7 \mathrm{f}$ & 250 & 250 & 500 & 250 & $>1000$ & $>1000$ & $>1000$ \\
\hline $8 \mathrm{a}$ & 125 & 200 & 250 & 125 & 500 & 500 & 500 \\
\hline $8 \mathrm{~b}$ & 200 & 250 & 125 & 125 & 500 & $>1000$ & $>1000$ \\
\hline $8 \mathrm{c}$ & 100 & 250 & 125 & 250 & 250 & 500 & 500 \\
\hline $8 \mathrm{~d}$ & 500 & 500 & 125 & 200 & $>1000$ & $>1000$ & $>1000$ \\
\hline $8 \mathrm{e}$ & 200 & 100 & 500 & 250 & $>1000$ & 250 & 500 \\
\hline $8 \mathrm{f}$ & 100 & 125 & 250 & 500 & 1000 & 1000 & 1000 \\
\hline Ampicillin & 100 & 100 & 250 & 100 & - & - & - \\
\hline Griseofulvin & - & - & - & & 500 & 100 & 100 \\
\hline
\end{tabular}

\section{Acknowledgment}

We are grateful to B. K. M. Science College, Valsad for providing research facilities, Atul Ltd. (Atul) for the IR spectral analysis, RSIC Punjab University for the ${ }^{1} \mathrm{H}$ NMR spectral analysis and D. Rajani, Microcare Laboratory, Surat, for antimicrobial activity screening.

\section{References}

1. Baradia R and Rao J T, Asian J. Chem., 2004, 16, 1194.

2. Hussain M A, Indian J. Chem., 2001, 40B, 324.

3. Yamakawa T, Kagechika H, Kawachi E, Hashimoto Y and Shudo K, J. Med. Chem., 1990, 33, 1430.

4. Rao M N, Naido L and Ramanan P N, Pharmazie, 1991, 46, 542.

5. Ninomio Y, Shimma N and Ishitsuka H, Antiviral Res., 1990, 13, 61 .

6. Methew J, Subba Rao A V and Rambhav S, Curr. Sci., 1984, 53, 576. 
7. Brzozowski Z, Kaminski Z and Angielski S, Acta Pol. Pharm., 1979, 36(6), 645 , Chem. Abstr., 1980, 93, 204525e .

8. Thron G D, Phytopathology, 1961, 51, 77 , Chem. Absrt., 1961, 55, 13749 i.

9. Fauran C, Turin M, Raynaud G and Pourrias B, (Delalande S. A) Fr. Demande 2,259,590 (C1. A61K, C07D), 29 Aug (1975), Appl. 74 03,681, 04 Feb (1974), 7pp, Chem. Abstr., 1976, 84, 59477y.

10. Mishriky N, Asad F M, Ibrahim Y A and Girgis A S, Indian J. Chem., 1996, 35B, 935.

11. Zidermane A, Duburs G, Zilbere A, Verpele R, Uldrikis J and Kumsars K, Latv. PSR Zinat. Akad. Vestis, 1991, 4, 77; Chem. Abstr., 1971, 75, 47266e.

12. Portnyagina V. A. and Danilenko V. K., Khim-Farm. Zh., 1971, 5(4), 15 ; Chem. Abstr., 1971, 75, 5843k .

13. Kuki M, Sakamoto Y and Ota Y, (Nippon Shoji K. K), Jpn Kokai Tokkyo Koho JP 04 95,078 [92 95, 078] (C1. C07D239/47), 27 Mar (1992), Appl. 90/210, 640, 08 Aug (1990); 8 pp; Chem. Abstr., 1992, 117, 131220d .

14. Weinhardt K K and Marx M, [syntax (U.S.A.); Inc.] Eur. Pat. Appl. 24,776 (C1. C07D239/12), 11 Mar (1981), US Appl. 71,442, 31 Aug (1979); 92 pp; Chem. Abstr., 1981, 95, 97837a.

15. Solankee A, Kapadia K, Thakor I, Patel J and Lad S, Orient. J. Chem., 2003, 19(3), 637; Chem. Abstr., 141, 2004, 260672x .

16. Solankee A, Kapadia K, Ciric A, Sokovic M, Doytchinova I and Geronikaki A, Eur. J. Med. Chem., 2010, 45(2), 510.

17. Solankee A, Patel K and Patel R, Der Chemica Sinica, 2011, 2(5), 1-7.

18. Rattan A, In: Antimicrobials in Laboratory Medicine. $5^{\text {th }}$ ed. B.Y. Churchill Livingstone, New Delhi, 2005, 85-90. 


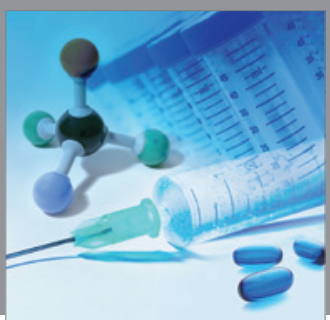

International Journal of

Medicinal Chemistry

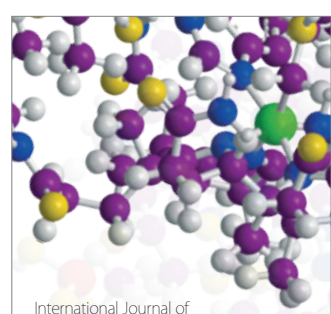

Carbohydrate Chemistry

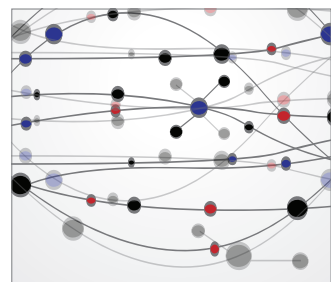

The Scientific World Journal
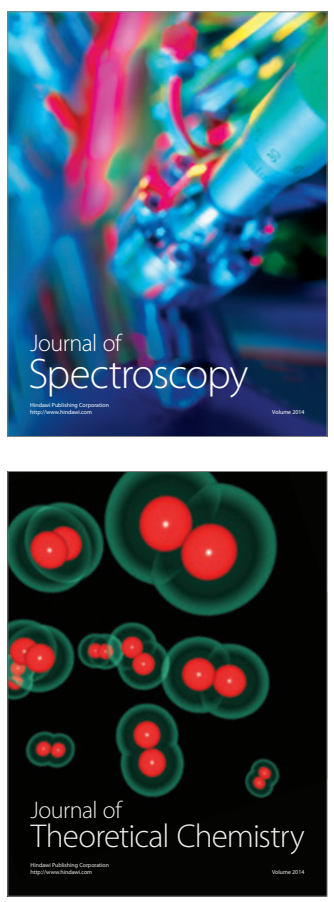
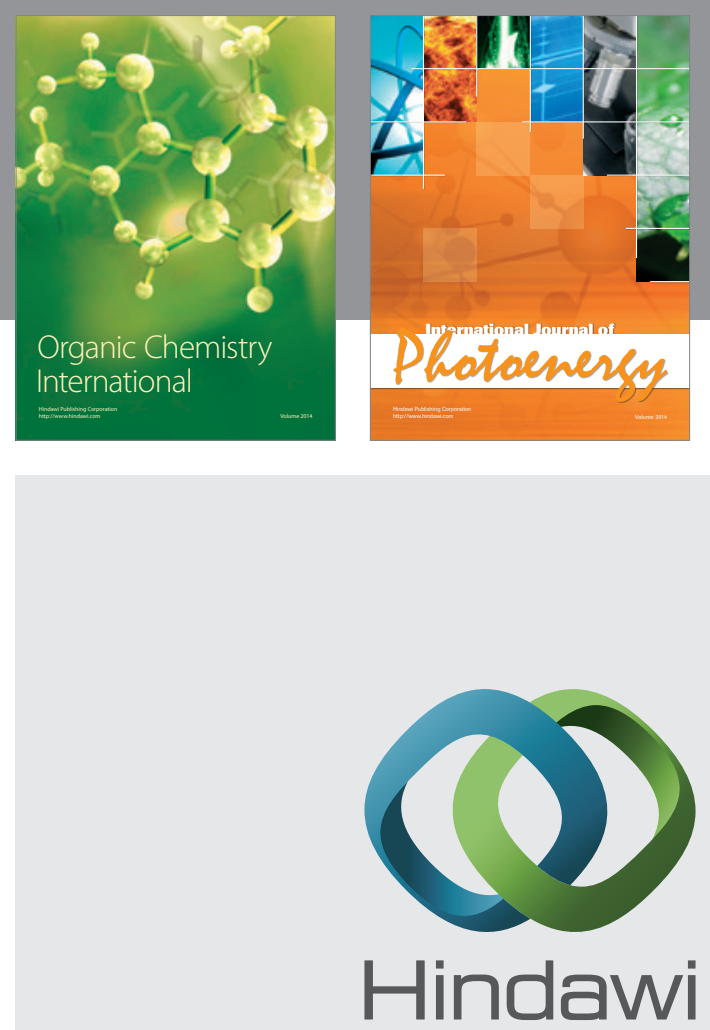

Submit your manuscripts at

http://www.hindawi.com
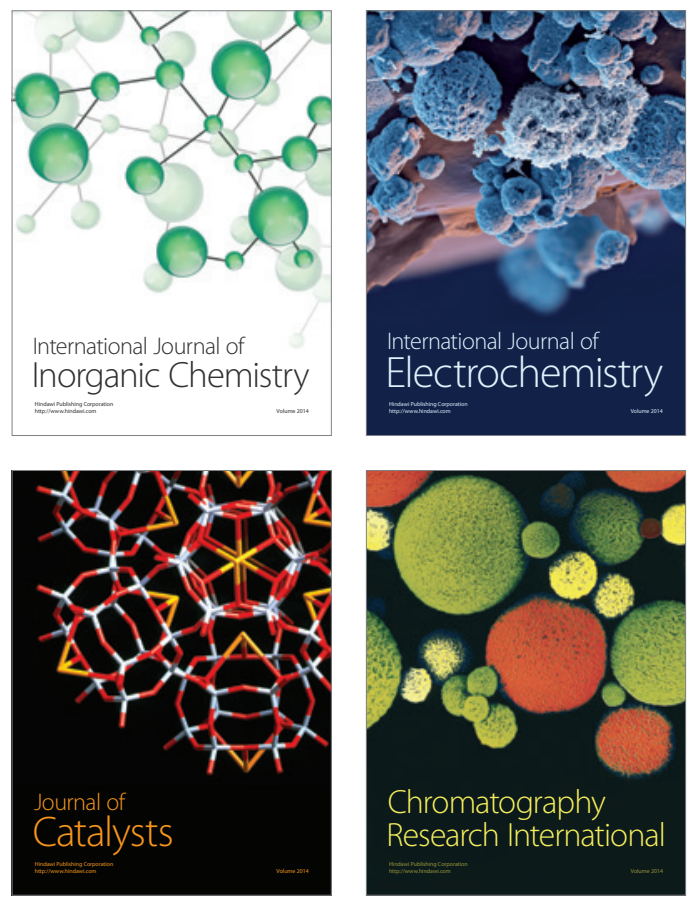
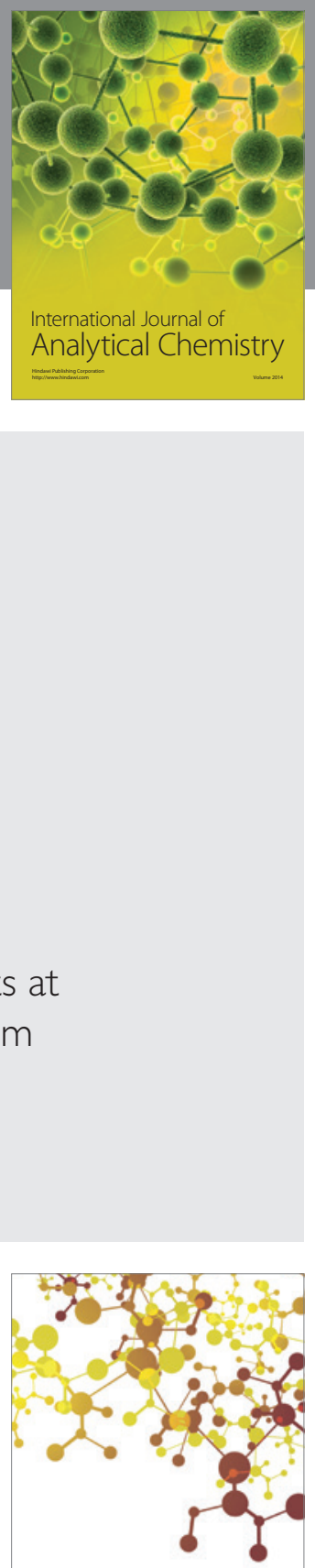

Journal of

Applied Chemistry
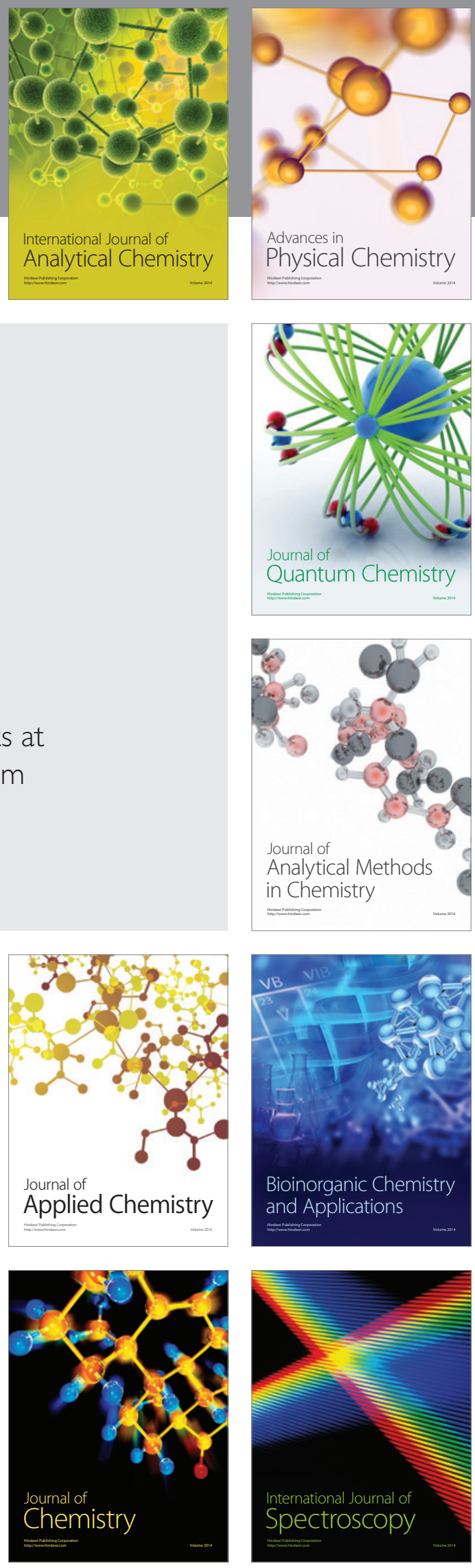